\title{
Kinetic study of velocity distributions in nanoscale semiconductor devices under room-temperature operation
}

\author{
N. Sano ${ }^{a)}$ \\ Institute of Applied Physics, University of Tsukuba, 1-1-1 Tennoudai, Tsukuba, Ibaraki, 305-8573 Japan
}

(Received 18 June 2004; accepted 14 September 2004)

\begin{abstract}
Quasiballistic electron transport in nanoscale semiconductor structures is investigated to clarify the importance of scatterings under room-temperature operation as reflected in the velocity distribution functions. The analyses are carried out for $n^{+}-n-n^{+}$structures based on the semiclassical Boltzmann transport equation (BTE). It is shown that the number of electrons with negative velocity grows exponetially due to scatterings around the top of the electronic potential barrier in the channel region and, thus, the scatterings cannot be neglected even in nanoscale device structures. This is closely related to the mathematical structure of the BTE whose solution exhibits the boundary-layer structure. (C) 2004 American Institute of Physics. [DOI: 10.1063/1.1812812]
\end{abstract}

The channel length in the state-of-the-art metal-oxidesemiconductor field effect transistors (MOSFETs) now reaches the order of tens of nanometers which is nearly comparable to or even smaller than a typical mean free path at room temperature. Electron transport in such small transit regions is, therefore, inherently nonstationary. Ballistic transport may be considered to be the extreme case of such nonstationary transport under strongly inhomogeneous structures. Hence, the interests of ballistic transport under such small structures, whose possibility was, however, pointed out more than two decades ago, ${ }^{1}$ have been revived recently as "ballistic MOSFETs." ${ }^{-4}$ In ballsitic MOSFETs, the channel electrons including hot electrons backscattered from the drain are assumed to cross the active region without scattering. The drain current is then evaluated by counting the right- and left-moving electrons at the electronic potential barrier formed near the source. ${ }^{5}$ We denote it hereafter as the ballistic theory. Therefore, the shape of the electron distribution function at the potential barrier plays a crucial role in the current evaluation. In the ballistic theory, the distribution function is assumed to be the combination of cold Maxwell distributions at the source and drain because of completely scattering-free channel.

In reality, the channel could never be scattering free even under undoped channels because of defects and/or surface roughness. In addition and probably more importantly, energy dissipation is inevitable in the analyses of irreversible transport characteristics. ${ }^{6}$ Therefore, transport properties in conventional devices have been customarily analyzed by solving the kinetic equation such as the Boltzmann transport equation (BTE). Recently, the effect of scatterings on ballistic transport properties in nanoscale device structures has been studied by numerically solving the nonequilibrium Green's function with a somewhat phenomenological scattering model. ${ }^{7,8}$ It has been pointed out that the scattering greatly affects the current, although the details of how the velocity distribution is different from that assumed in the ballistic theory are not clarified. In fact, the significance of scatterings in electron transport under small device structures had been already recognized by Baranger and Wilkins long before ballistic MOSFETs became the subject. ${ }^{9}$ They have

${ }^{a)}$ Electronic mail: sano@esys.tsukuba.ac.jp solved the BTE coupled self-consistently with the Poisson equation for $n^{+}-n-n^{+}$structures and found that the velocity distribution function is greatly broadened due to scatterings. The device size considered in their work was, however, too large to expect the ballistic limit. Furthermore, the physical origin of the broadening in the velocity distribution function was not clearly resolved.

In the present letter, the velocity distribution function at the top of the electronic potential barrier in the channel is explicitly obtained by solving the BTE and directly compared with that assumed in the ballistic theory. It is shown that the velocity distribution function obtained from the BTE always exhibits non-negligible electrons with negative velocity at the top of the electronic potential barrier and that the assumption of the scattering-free channel is inappropriate even in nanoscale semiconductor structures. It is pointed out that this is closely related to the mathematical structure of the BTE whose solution exhibits the boundary-layer structure.

In order to avoid the complications associated with the geometry, the sample structure we consider is a onedimensional $n^{+}-n-n^{+}$diode, which consists of a lightly doped thin slab (we denote it by channel) sandwiched with two highly doped thick regions (we denote them by $\mathrm{S}$ and $\mathrm{D}$ regions). Figure 1 shows the schematic diagram of an

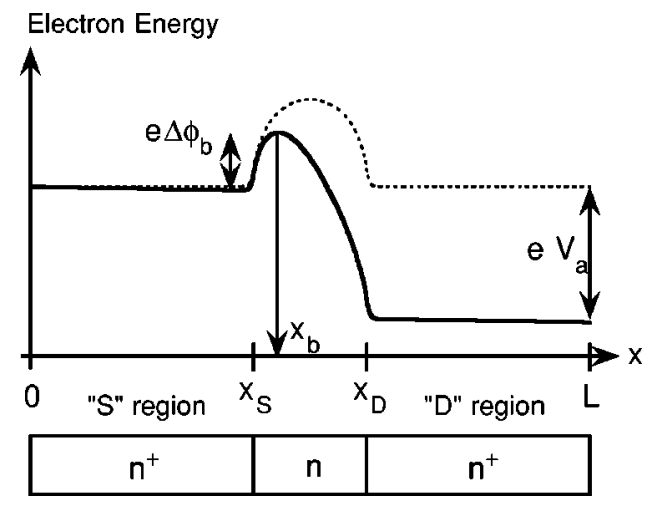

FIG. 1. Schematic diagram of an $n^{+}-n-n^{+}$diode employed in this study and a typical energy diagram for electrons under an applied voltage $V_{a}$. The position of the edges of highly doped ( $\mathrm{S}$ and $\mathrm{D}$ ) regions is denoted as $x_{S}$ and $x_{D}$, respectively. The position of the top of the potential barrier for electrons is denoted by $x_{b}$. 
$n^{+}-n-n^{+}$diode and a typical energy diagram under an applied voltage $V_{a}$. We suppose that the donor densities $N_{D}(x)$ in the S/D and channel regions are $10^{20}$ and $10^{18} \mathrm{~cm}^{3}$, respectively. In addition, the electrons in the S/D regions are treated as nondegenerate for the sake of simplicity. The length of the highly doped regions is chosen long enough, compared with the relaxation length, for the electron distribution function to relax into the quasithermal equilibrium distribution.

Because the temperature is so high that the phaserandomizing scattering is inevitable (as we shall demonstrate in this study), the present analyses are based on the semiclassical BTE. We have followed the approach taken by Baranger and Wilkins ${ }^{9}$ with a few changes; We first solve the Poisson equation and the drift-diffusion equation self-consistently. ${ }^{10}$ The BTE is then solved under the electrostatic potential $V(x)$ obtained from the drift-diffuion calculations. The collision integral is treated with the relaxation time approximation and, thus, the BTE under steady state is given by

$$
v \frac{\partial f(x, v)}{\partial x}+\frac{e}{m^{*}} \frac{d V(x)}{d x} \frac{\partial f(x, v)}{\partial v}=-\frac{f(x, v)-f_{\mathrm{eq}}(x, v)}{\tau(x)},
$$

where $v$ is the velocity, $m^{*}\left(=0.322 m_{e}, m_{e}\right.$ : electron mass $)$ is the effective mass, $e$ is the magnitude of electronic charge, $\tau(x)$ is the relaxation time, and $f_{\mathrm{eq}}(x, v)$ is the local equilibrium distribution function given by the product of the electron density $n(x)\left[=\int d v f(x, v)\right]$ and the Maxwell distribution. Notice that the relaxation time $\tau(x)$ in Eq. (1) is introduced as a function of position $x$. In the present calculations, the relaxation time for the channel region is extracted from a typical mobility $\mu_{n}\left[=e \tau(x) / m^{*}\right]$ for bulk Si with $N_{D}$ $=10^{18} \mathrm{~cm}^{-3}$ at $T=300 \mathrm{~K}$ that is about $270 \mathrm{~cm}^{2} / \mathrm{V} \mathrm{s}^{11}$ and, thus, assumed to be $50 \mathrm{fs}$. The relaxation time in highly doped S/D regions where the short-range electron-electron scattering is most effective in relaxing the nonequilibrium electron distribution functions is assumed to be $18 \mathrm{fs}$, which is equivalent to $\mu_{n}=100 \mathrm{~cm}^{2} / \mathrm{V} \mathrm{s}$.

The current in the ballistic limit is evaluated by the number of electrons passing through the top of the electronic potential barrier $\left(x=x_{b}\right)$ per unit time by assuming that the electron distribution functions at $x=x_{S}$ and $x_{D}$ are given by the cold Maxwellians. Therefore, how close the device is to the ballistic limit may be measured by comparing the averaged electron velocity $v_{\text {kin }}$ evaluated from the BTE with that $v_{\text {ball }}$ evaluated from the ballistic theory at the top of the potential barrier $\left(x=x_{b}\right)$. Figure 2 shows the ratio $v_{\text {kin }} / v_{\text {ball }}$ as a function of the channel length $L_{c}$ under the applied voltages $V_{a}=0.1,0.2,0.3$, and $0.4 \mathrm{~V}$. For comparison, the exponential dependence of the ratio $\left[\propto \exp \left(-\alpha L_{c}\right)\right.$ with $\alpha=$ constant] is also plotted with a dashed line. Indeed, the ratio exponentially approaches the limit $\left(v_{\text {kin }} / v_{\text {ball }}=1\right)$, as $L_{c}$ shrinks. However, we would like to stress that in spite of the fact that the ratio is close to the ballistic limit, the physics behind the electron transport is rather different from the simple story based on the ballistic theory, as we show below.

Figure 3 shows a typical result of the electron distribution function $f(x, v) / n(x)$ at various positions in the $n^{+}-n-n^{+}$diode $\left(L_{c}=15 \mathrm{~nm}\right)$ as a function of velocity normalized with the thermal velocity $v_{\text {th }}\left[=\sqrt{k_{B} T / m^{*}}, k_{B}\right.$ : the Boltzmann constant, $T(=300 \mathrm{~K})$ : the lattice temperature]. Since the electron mean free path in the channel region is around $10 \mathrm{~nm}$, the ballistic electrons dominate in the shape of the Downloaded 21 Mar 2007 to 130.158.56.229. Redistribution subject

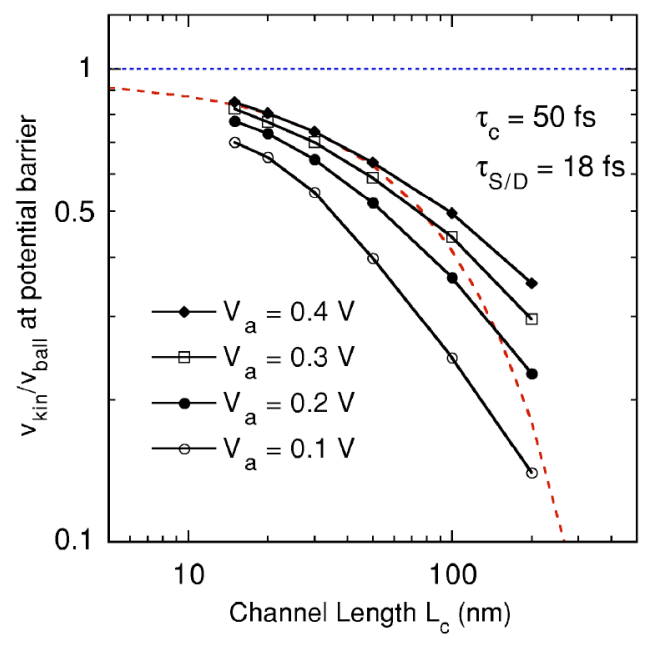

FIG. 2. Channel length dependence of the ratio $v_{\text {kin }} / v_{\text {ball }}$ under $V_{a}$ $=0.1,0.2,0.3,0.4 \mathrm{~V}$. The dashed line represents the exponential dependence of the ratio $\left[\propto \exp \left(-\alpha L_{c}\right)\right]$. The relaxation times in the channel and $\mathrm{S} / \mathrm{D}$ regions are assumed to be 50 and $18 \mathrm{fs}$, respectively.

distribution functions. The distribution function is, however, deformed as soon as electrons pass over the potential barrier $\left(x_{b}=53 \mathrm{~nm}\right)$. In other words, the ballistic peak is not isolated from the other velocity components and the distribution function is greatly broadened. This broadening is due to scatterings, although the electron's path is much smaller than the mean free path. It is interesting to note that the distribution functions in Fig. 3 turn out to be very similar in shape to those of Fig. 3 in Ref. 9, although the size of the present diode is smaller by a factor of 27 .

Figure 4 is our main result and shows the velocity distribution functions obtained from the BTE at the top of the electronic potential barrier $\left(x=x_{b}\right)$ with various channel lengths $\left(L_{c}=50,30,15 \mathrm{~nm}\right)$. The equilibrium Maxwell distribution and the distribution function assumed in the ballistic theory are also plotted with the dashed and dotted curves, respectively. Notice that the distribution function under the ballistic limit is given by the hemi-Maxwellian and, thus, the number of electrons coming ballistically from the D (right) region is negligible. On the other hand, the velocity distribu-

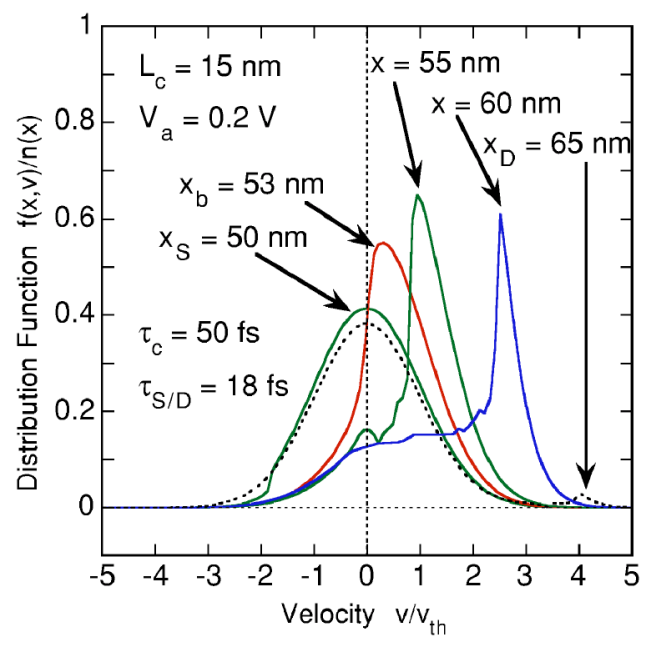

FIG. 3. Electron distribution functions $f(x, v) / n(x)$ at various positions $(x$ $=50,53,55,60,65 \mathrm{~nm})$ as a function of the normalized velocity $v / v_{\text {th }}$. The channel length $L_{c}\left(=x_{D}-x_{S}\right)$ is $15 \mathrm{~nm}$ and $V_{a}=0.2 \mathrm{~V}$. The top of the potential

barrier is located at $x_{b}=53 \mathrm{~nm}$.
to AIP license or copyright, see http://apl.aip.org/apl/copyright.jsp 


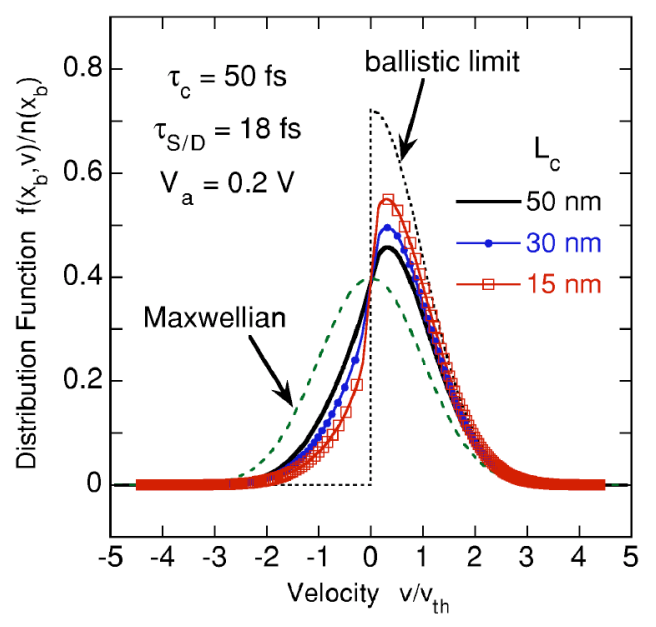

FIG. 4. Velocity distribution functions obtained from the BTE at the top of the potential barrier $\left(x=x_{b}\right)$ for $L_{c}=50,30,15 \mathrm{~nm}$ under $V_{a}=0.2 \mathrm{~V}$. The dashed and dotted curves represent the equilibrium Maxwellian and the distribution function under the ballistic limit, respectively.

tion obtained from the BTE always exhibits non-negligible electrons with negative velocity. Under the present framework of the relaxation time approximation, the negativecomponent velocity at the top of the potential barrier is generated from the electrons' flow from the D (right) region. Since the number of electrons coming ballistically from the $\mathrm{D}$ region is negligible, these electrons result from scatterings in the channel region. We have found that the electrons with negative velocity are not negligible even when the relaxation time in the channel is longer than $200 \mathrm{fs}$, which is much longer than the transit time over the channel region. The broadening observed in the distribution functions of Fig. 3 has also the same origin.

This finding is closely related to the mathematical structure of the BTE; changing the variables from $(x, v)$ to $(x, \varepsilon)$ with $\varepsilon=m^{*} v^{2} / 2-e V(x)(\varepsilon \geqslant 0)$, Eq. (1) is expressed as

$$
\pm \sqrt{\frac{2}{m^{*}}\{\varepsilon+e V(x)\}} \frac{d f(x, \varepsilon)}{d x}=-\frac{f(x, \varepsilon)-f_{\mathrm{eq}}(x, \varepsilon)}{\tau(x)},
$$

where $+(-)$ sign corresponds to the positive (negative) component of electron velocity. Therefore, the BTE close to the band edge around the top of the potential barrier becomes singular and its solution exhibits the boundary-layer structure, in which the solution of the differential equation changes very rapidly. ${ }^{12}$ The ballistic transport is just a solution in the outer region where the distribution function changes slowly and, thus, it breaks down in the boundary layer region. This could be physically interpreted as follows. When electrons pass around the top of the potential barrier, their velocities could be indefinitely small. As a result, electrons spend longer time around the potential barrier and suffer scatterings. These electrons are, therefore, agitated and thermalized so the number of electrons with negative velocity grows exponentially. Notice that they are not just the backscattered electrons injected from the S (left) region, but rather they are the whole electrons with energy close to the band edge residing around the top of the potential barrier. This is why the velocity distribution obtained from the BTE always shows non-negligible electrons with negative velocity. Full discussion on this point will be presented elsewhere with mathematical details. ${ }^{13}$

In summary, we have investigated electron transport in nanoscale semiconductor structures by employing the the semiclassical BTE. The direct comparison of the velocity distribution functions obtained from the BTE with those of the ballistic theory has been made. It has been shown that the ballistic electrons are indeed crucial, but the scattering in the channel region cannot be neglected due to the boundarylayer structure in the solution of the BTE.

${ }^{1}$ M. S. Shur and L. F. Eastman, IEEE Trans. Electron Devices ED-26, 1677 (1979); Solid-State Electron. 24, 11 (1981).

${ }^{2}$ M. Lundstrom, IEEE Electron Device Lett. EDL-18, 361 (1997).

${ }^{3}$ F. G. Pikus and K. K. Likharev, Appl. Phys. Lett. 71, 3661 (1997); Y. Naveh and K. K. Likharev, IEEE Electron Device Lett. EDL-21, 242 (2001).

${ }^{4}$ M. Lundstrom, IEEE Trans. Electron Devices ED-49, 133 (2002).

${ }^{5}$ K. Natori, IEICE Trans. Electron. E-84C, 1029 (2001).

${ }^{6}$ For example, R. Balescu, Statistical Dynamics (Imperial College Press, New York, 1997).

${ }^{7}$ M. P. Anantram and A. Svizhenko, IEEE Trans. Electron Devices ED-50, 1459 (2003).

${ }^{8}$ R. Venugopal, M. Paulsson, S. Goasguen, S. Datta, and M. Lundstrom, J. Appl. Phys. 93, 5613 (2003).

${ }^{9}$ H. U. Baranger and J. W. Wilkins, Phys. Rev. B 30, 7349 (1984).

${ }^{10} \mathrm{~S}$. Selberherr, Analysis and Simulation of Semiconductor Devices (Springer, Berlin, 1984).

${ }^{11}$ R. F. Pierret, Semiconductor Fundamentals (Addison-Wesley, New York, 1988), p. 66.

${ }^{12}$ C. M. Bender and S. A. Orszag, Advanced Mathematical Methods for Scientists and Engineers (McGraw-Hill, New York, 1978).

${ }^{13} \mathrm{~N}$. Sano (unpublished). 